\title{
Treatment of scalp lacerations with a hair apposition technique reduced scarring, pain, and procedure duration compared with suturing
}

\author{
Ong Eng Hock M, Ooi SB, Saw SM, et al. A randomized controlled trial comparing the hair apposition technique with \\ tissue glue to standard suturing in scalp lacerations (HAT study). Ann Emerg Med 2002;40:19-26.

\section{QUESTION: Is a new technique of treating scalp lacerations (the hair apposition technique [HAT]) as effective as standard suturing for wound healing, complications, and pain?}

Source of funding:

tissue adhesive

(Histoacryl Blue)

provided by B Braun

(Melsungen, Germany).

For correspondence:

Dr M E H Ong,

Singapore General

Hospital, Singapore.

gaeoeh@sgh.gov.sg

Abstract and

commentary also

appear in

Evidence-Based

Nursing.

\section{Design}

Randomised (allocation concealed*), unblinded,* controlled trial with follow up at 1 week.

\section{Setting}

The emergency departments (EDs) of 2 tertiary care hospitals in Singapore.

\section{Patients}

189 patients of any age (mean age $32 \mathrm{y}, 74 \%$ men) with linear non-stellate lacerations of the scalp that were $<10 \mathrm{~cm}$ in length and with scalp hair $>3 \mathrm{~cm}$ in

Hair apposition technique (HAT) v standard suturing (SS) for treatment of scalp lacerations in the emergency department $\dagger$

\begin{tabular}{lllll} 
Outcomes at 1 week & HAT & SS & RRR (95\% CI) & NNT (Cl) \\
Any complication & $7.4 \%$ & $21.5 \%$ & $66 \%(25$ to 85$)$ & 8 (5 to 24$)$ \\
\hline Scarring & $6.3 \%$ & $20.4 \%$ & $69 \%(29$ to 87$)$ & 8 (5 to 22$)$ \\
\hline
\end{tabular}

†Abbreviations defined in glossary; RRR, NNT, and $\mathrm{Cl}$ calculated from data in article.

\section{COMMENTARY}

Treatment of lacerations can be a traumatic experience for patients, especially children. Ong et al evaluated an alternative treatment for scalp laceration that is painless and innovative. The advantages of the HAT are that it is time efficient and can be used by all levels of staff.

The HAT may be limited in the type of scalp laceration on which it can be used. The authors noted, for example, that the HAT was not used for bleeding wounds because the technique does not induce haemostasis. In the study, the mean laceration length was 3 $\mathrm{cm}$, but the depth or width of lacerations was not reported. Clinicians should be careful when considering using the technique for deep wounds, as it is designed to allow apposition of the outermost layers of skin, and in the case of deep scalp wounds, this could result in complications such as haematoma formation, infection, dehiscence, and increased scarring. ${ }^{1}$

Assessments were completed at 1 week, and follow up was only continued for patients who had complications at this assessment. Longer term outcomes of lacerations include functionality and cosmetic appearance. Short term follow up was probably appropriate in this trial, as functionality and cosmetic appearance would be relatively unimportant with most scars being covered by hair. ${ }^{1}$

A recent Cochrane Collaboration review on tissue adhesives for traumatic laceration found a small but significant increase in the rate of dehiscence when tissue adhesives were used to close wounds. ${ }^{2}$ This highlights the question of whether use of the HAT with tissue adhesives in scalp lacerations may reduce the risk of dehiscence more than the use of tissue adhesives alone. A further trial comparing the HAT plus tissue adhesives with tissue adhesives alone for scalp lacerations is warranted and would assist decision making around treatment of scalp lacerations. An economic evaluation comparing standard intervention with tissue adhesives (with or without the HAT) would also be of value.

Jane Brosnahan, RCPN, BN

Centre for Evidence Based Nursing Aotearoa Auckland District Health Board Auckland, New Zealand

1 Singer AJ. Hair apposition for scalp lacerations. Ann Emerg Med 2002;40:27-9.

Farion $\mathrm{K}$ Osmond $\mathrm{MH}$, Hartling $\mathrm{L}$ et al Tissue adhesives for traumatic lacerations in children and adults. Cochrane Database Syst Rev 2002;(3):CD003326.

length. Exclusion criteria were severely contaminated wounds, actively bleeding wounds that did not stop bleeding after $\geq 5$ minutes of applied pressure, and unstable vital signs or neurological status requiring priority resuscitation. 188 patients $(99 \%)$ were included in the analysis.

\section{Intervention}

96 patients were allocated to the HAT. Wounds were cleansed according to standard procedure, and no local anaesthetics were given. The wound was closed by bringing together the hair on both sides of the wound and making a single twist; no actual knot was made. The twist was then secured with tissue glue. Patients were instructed to wash their hair after the third day and told that the glue would gradually fall off. 93 patients were allocated to standard suturing, whereby the wound was cleansed, an injection of local anaesthetic was given, and hair was shaved or trimmed according to local practice. The wound was then sutured, and removal of sutures was done 1 week later. Hair washing was discouraged for 1 week. Oral sedation was sometimes required for very young children.

\section{Main outcome measures}

Main outcomes were complications (infection, scarring, bleeding, and wound breakdown) and satisfactory wound healing. Secondary outcomes included pain (10 point visual analogue scale with 0 being no pain) and duration of the procedure.

\section{Main results}

Analysis was by intention to treat. At 1 week, fewer patients in the HAT group than in the standard suturing group had any complications or had scarring (table); the groups did not differ for infection $(1.1 \% v 1.1 \%, \mathrm{p}=1.0)$, bleeding $(0 \% v 1.1 \%, \mathrm{p}=0.49)$, wound breakdown $(0 \% v$ $4.3 \%, \mathrm{p}=0.057)$, or satisfactory wound healing $(100 \% v$ $96 \%, \mathrm{p}=0.057$ ). Patients who received the HAT reported less pain than those who had standard suturing (median score $2 v 4$ out of $10, \mathrm{p}<0.001)$. The HAT was performed more quickly than standard suturing (median $5 v 15$ min, $\mathrm{p}<0.001$ ).

\section{Conclusion}

Treatment of scalp lacerations using a hair apposition technique reduced scarring, overall complications, pain, and procedure duration compared with standard suturing, but did not differ for satisfactory wound healing, infection, bleeding, or wound breakdown.

*See glossary. . 